\title{
MULTI-COMPONENT SPATIAL OPTICAL SOLITONS IN PHOTOREFRACTIVE MEDIUM
}

\author{
W. KRóLIKowsKi* \\ Australian Photonics CRC, Laser Physics Centre \\ Australian National University, Australia
}

\begin{abstract}
We discuss properties of one- and two-dimensional multi-component spatial solitons. We present our recent results on formation and interaction of these solitons in photorefractive nonlinear crystal.

PACS numbers: 42.65.Tg, 42.65.Hw
\end{abstract}

A bright spatial optical soliton represents an optical beam propagating in a nonlinear medium without changing its intensity profile [1]. Since the condition for the soliton formation requires that the index of refraction of the medium increases with light intensity (the self-focussing effect), the presence of the soliton means the formation of the region of elevated refractive index which is nothing else than a waveguide. Therefore, a spatial optical soliton represents a self-trapped beam - a mode of the self-induced waveguide $[2,3]$. Typically, the soliton is just a fundamental mode of such waveguide. However, the optical waveguide can support, in general, not a single but many spatial modes. It is therefore natural to pose a question whether a few solitons can co-propagate in a stationary manner so, by the self-consistency, they will be simultaneously of higher order modes of the self-induced waveguide. It turns out that the answer to this question is positive. It has been demonstrated recently that superposition of multiple mutually temporally incoherent optical beams in a slow nonlinear medium results in the formation of a complicated soliton object called a multi-component (or vector) soliton [4]. However, the stationary propagation of such structure can only occur if the constituent modes do not interfere. This can be achieved in two different ways. Firstly, one may use beams of orthogonal polarization. Indeed such vector solitons have been already studied theoretically [5-7] and also observed experimentally [8]. The use of orthogonal polarizations however, limits the number of constituent components to two. Another option is to employ co-propagating mutually incoherent beams. In experimental conditions the mutual incoherence of optical beams is obtained by using a medium with a slow nonlinear response. Strictly speaking the constituent beams are not incoherent. The effective mutual incoherence is obtained by shifting the frequency of each beam by the amount much larger than the inverse of the

*e-mail: wzk111@rsphysse.anu.edu.au 
response time of the nonlinear medium. In this way all beams remain coherent but their relative phases experience fast temporal oscillations (determined by the detuning). If the nonlinearity is slow then it cannot follow these fast oscillations and will respond to the average intensity which will be given as a sum of intensities of all components.

The first experimental evidence of this type multi-component solitons has been presented by Princeton group [4]. In this case the photorefractive crystal (which exhibits a slow nonlinear response) served as a nonlinear medium. By using two or three mutually incoherent input beams the formation of single or multi-peak ("multi-hump") spatial solitons has been demonstrated.

Here in this paper some of the features of the multi-component spatial solitons will be discussed in a slow nonlinear medium.

If $\psi_{j}(x, y, z)$ represents a slowly varying amplitude of the $j$-th component of the composite beam propagating along $z$-axis, then its evolution can be described by the system of the coupled nonlinear Schrödinger equations [9]

$$
\mathrm{i} \frac{\partial \psi_{j}}{\partial z}+\frac{1}{2}\left(\frac{\partial^{2} \psi_{j}}{\partial x^{2}}+\frac{\partial^{2} \psi_{j}}{\partial y^{2}}\right)+F(I) \psi_{j}=0
$$

where $I=\sum_{j}\left|\psi_{j}\right|^{2}$ is the total light intensity. The function $F(I)$ represents a fenomenological model of the nonlinearity, and $F(I)=I$ in the case of Kerr medium. The stationary solution in the form $\psi_{j}=u_{j}(x, y) \exp \left(-\mathrm{i} \lambda_{j} z\right)$ transforms the system Eq. (1) into an eigenvalue problem

$$
\lambda_{j} u_{j}+\frac{1}{2}\left(\frac{\partial^{2} u_{j}}{\partial x^{2}}+\frac{\partial^{2} u_{j}}{\partial y^{2}}\right)+F(I) u_{j}=0 .
$$

The structure of Eq. (2) indicates that the multi-component soliton can be treated as a multi-mode self-induced waveguide with the refractive index profile given by the function $F(I)$ [10-12]. Each soliton component represents then a particular higher order mode of the waveguide with the corresponding propagation constant $\lambda_{j}$. It turns out that in the one-dimensional case and for Kerr nonlinearity, the system of Eqs. (1), (2) is completely integrable and its solution represents a propagation of the soliton complex consisting of many soliton components (or modes) [13-15]. The whole solution depends on $(2 N-1)$ parameters ( $N$ is the number of components) which leads to a huge diversity of the soliton shape. In general all these solitons have asymmetric and, often, multi-peak intensity profile. In Fig. 1 we show an example of the composite soliton consisting of two mutually incoherent components. They correspond respectively to the fundamental and first mode of the self-induced waveguide whose index profile is given by the total intensity (Fig. 1a).

It is well known that solitons of integrable systems exhibit particle-like properties. They are robust objects and their collisions are elastic — solitons preserve their structure in collisions. It appears that the multi-component solitons of Kerr nonlinearity exhibit quite surprising collisional properties, namely they reshape in collisions. In Fig. 2 we show an example of collision of solitons consisting of three incoherent components each. It is evident that the collision induces a dramatic change of shape of these solitons. After the collision each beam remains a soliton but has a different intensity profile. In this particular case single-peak 

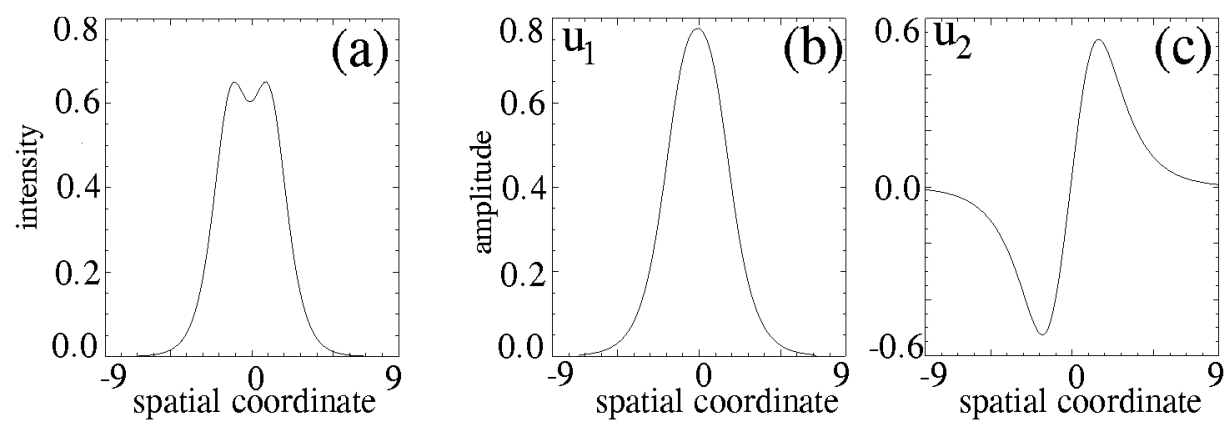

Fig. 1. Intensity (a) and amplitude (b-c) distribution of the two-component composite soliton in Kerr medium.

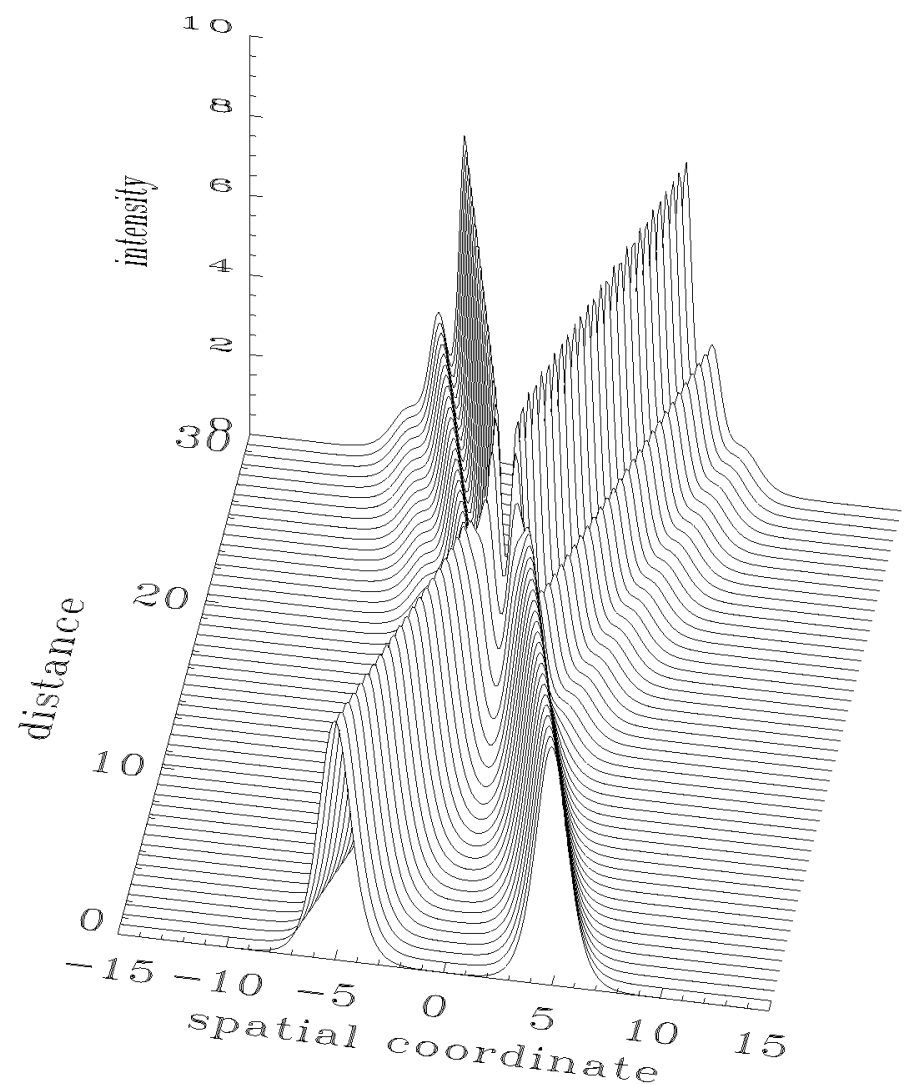

Fig. 2. Collision of identical three-component vector solitons in Kerr medium - numerical simulations.

solitons develop a three-peak structure upon the collision. This reshaping can be explained using the concept of the multi-soliton complex. It is well known that solitons collision in Kerr medium leads to a lateral shift of the solitons [16]. 
Each soliton component experiences the lateral shift which depends on the amplitude of the component and the relative angle of collision. Since components have different amplitudes their lateral shifts are also different. In effect the profile of the soliton changes after collision.

The collisional properties of multi-component solitons have been studied in experiments with photorefractive crystals [17]. It is well known that externally biased photorefractive crystal exhibits a strong positive or negative nonlinearity [18]. They have been used to demonstrate the formation of bright and dark spatial solitons. To study the formation and collision of multi-component solitons we used an experimental system as shown in Fig. 3. A laser beam $(\lambda=514.5 \mathrm{~nm})$ was split into two parts. The first one was reflected from an oscillating mirror $(\approx 1 \mathrm{kHz})$ which introduced a frequency shift into the beam. This frequency-shifted beam constituted the fundamental component of the input composite beam. A second, higher order component was created by introducing a glass slide across the half of the second beam. After adjusting the phase across the beam (by tilting the glass slide) to $\pi$ the structure of the beam resembled that of a first-order mode of the planar waveguide. Both beams were then recombined and focused by a set of cylindrical lenses to form a narrow $(\approx 20 \mu)$ stripe on the input face of the crystal.

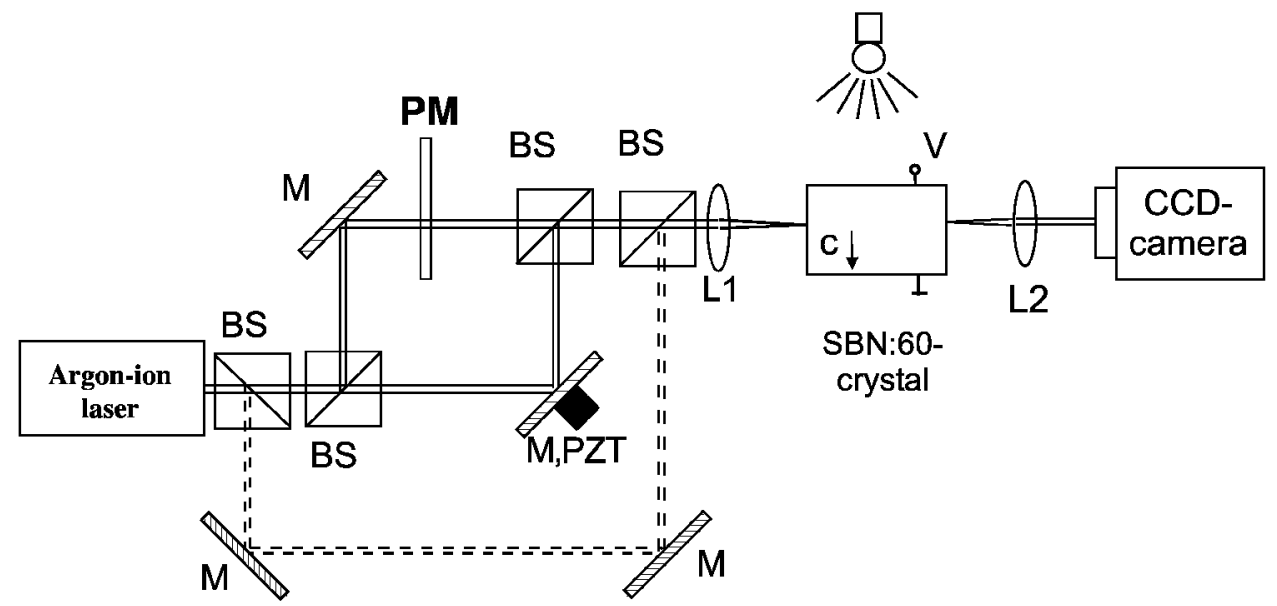

Fig. 3. Experimental setup used to study the formation and collision of multi-component solitons. L - lens, M - mirror, BS - beam splitter, PM - phase mask, PZT - piezo-electric transducer. The dashed line denotes a path of the beam forming a fundamental soliton which intersects in the crystal with a composite soliton.

The composite beam then intersected inside the crystal with a single component stripe beam derived from the same laser. The optical path for this second beam exceeded that of the first one by a few coherence lengths so both beams were mutually incoherent. The propagation distance inside the crystal was $10 \mathrm{~mm}$. The output face of the crystal was imaged onto a CCD camera and recorded by the computer. The crystal was biased with a $1.5 \mathrm{kV}$ dc electric field applied along its optical axis while all beams were extraordinarily polarized to make use of the largest $r_{33}$ electro-optic coefficient. Additionally, the crystal was illuminated by 
the broad white light beam which provided the necessary background illumination used to control the degree of saturation of the nonlinearity which in our experiments was estimated to be of the order of unity.

In Fig. 4 we present an example of the collision of the two-component soliton and the fundamental soliton. This graph shows the intensity distribution (and its profile) as seen at the output face of the strontium-barium-niobate (SBN) crystal. The pictures in the top row ( $a-b$ ) illustrate the free propagation of the single-peak (a) and two-hump (b) soliton while that in the bottom row show the effect of the collision. It is clear that the collision results in a strong change of the intensity profile of the composite beam which develops a strongly asymmetric structure.
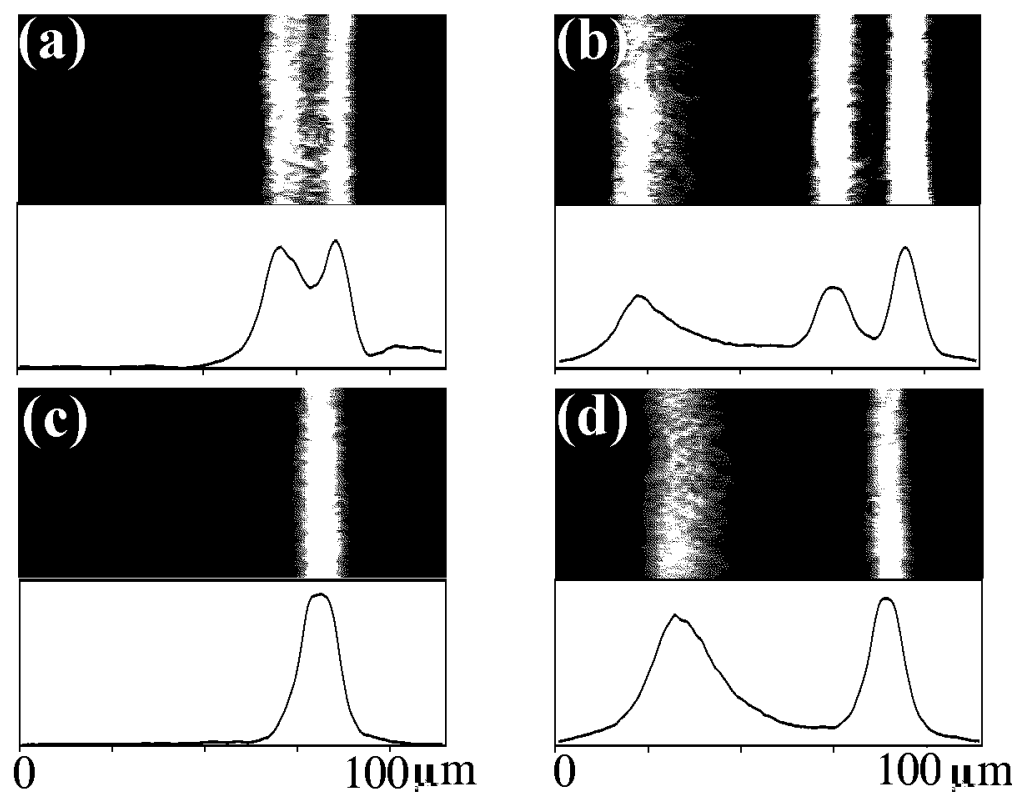

Fig. 4. Collision of 1-dimensional two-component and fundamental solitons in photorefractive crystal; ( $\mathrm{a}-\mathrm{b})$ intensity distribution of the two-component soliton before the collision; (c-d) results of the collision with the fundamental soliton. Parameters are: $V=1.5 \mathrm{kV}$, the propagation distance $z=10 \mathrm{~mm}$.

Although the experimental observations seem to confirm collisional properties of the multi-component solitons, it is important to realize that, while photorefractive nonlinearity resembles Kerr-type for low light intensity, it saturates for high intensity. In the 1-dimensional case nonlinearity can be expressed as $F(I) \propto 1 /(1+I)[18]$. For the saturable nonlinearity the system of Eqs. (1) is no longer integrable. This has profound consequences on the properties of multi-component solitons. Firstly, it turns that among all stationary solutions only the symmetric solutions are stable. Although the asymmetric solutions are still possible [19] and may propagate over a distance of a few diffraction lengths they will finally transform into symmetric solitons. Secondly, a collision of solitons 
in saturable medium is no longer elastic. Our numerical simulations show that the collision still induces a shape change of the soliton [17]. However, as this structure no longer corresponds to the stationary solution, it exhibits mutual spatial oscillations of the constituent components. In effect the soliton intensity profile varies in propagation. Therefore, it appears that the results shown in Fig. 4 actually illustrate one particular stage of the oscillation of the soliton intensity profile. To check this hypothesis one would have to monitor the soliton structure as it propagates from the collision region through the crystal. This requires a wedge-cut crystal which allows for variation of the propagation distance [20]. In our experiments we used a crystal of fixed length and hence had to resort to an indirect method. We fixed all parameters of colliding fundamental and two-component solitons as well as their interaction angle but varied the location of the collision region inside the crystal. This was done by parallel shifting of the fundamental beam (see Fig. 5a). The output intensity distribution (and corresponding intensity profile) for a few different values of the collision distance (measured from the front facet of the crystal) are shown in Fig. 5b. The graph in the top row illustrates the no-collision event. The composite soliton exhibits a well-defined two-peak structure. The subsequent graphs display the result of the collision as the impact region moves from the front towards the exit face of the crystal. It is clear that the transverse structure of the vector soliton strongly changes (compare the relative height and location of the peaks of the composite soliton) with the distance from the collision region to the exit face of the crystal. This behaviour is in accordance with numerical simulations which show the collision-initiated oscillations of the soliton structure [17].

(a)

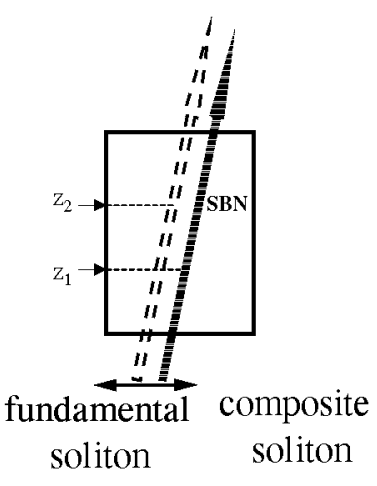

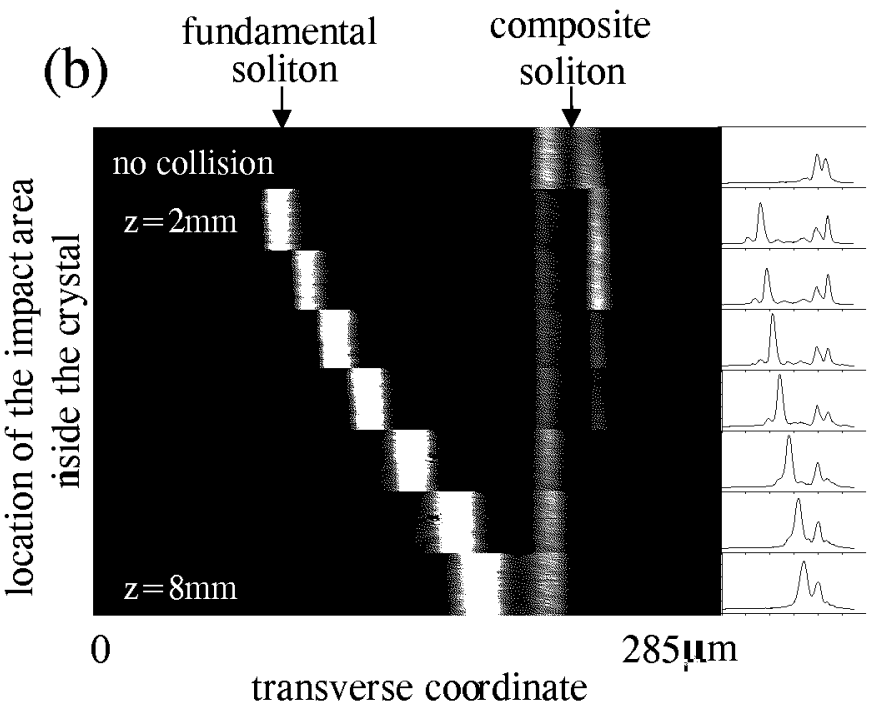

Fig. 5. Collision of composite and fundamental solitons as a function of location of impact area in the crystal; (a) schematic of the interaction; (b) light intensity distribution (and its profile) at the exit face of the crystal. Experimental parameters are the same as in Fig. 4. 
It is worth to mention that recently the theory of 1-dimensional composite solitons has been further advanced to describe the effect of simultaneous presence of both mutually incoherent and coherent soliton components [21].

The concept of the above-described 1-dimensional multi-component solitons can be extended to two-dimensional structures. These are the so-called dipole-mode vector soliton, and has been predicted theoretically very recently [22]. It is a novel type of an optical vector soliton that originates from trapping a dipole $\mathrm{HG}_{01}$-type mode in one optical beam by a fundamental-soliton-induced waveguide created by the other, co-propagating beam. Moreover, it has been shown that, while many other topologically complex structures may be created, it is only the dipole mode that is expected to generate a family of dynamically robust vector solitons. For instance, the recently studied vortex-mode vector soliton, which has a node-less shape beam in one component and an optical vortex, i.e. beam with phase singularity [23] in the other component, undergoes a symmetry-breaking instability which transforms it into a radially asymmetric dipole-mode vector soliton, even in a perfectly isotropic nonlinear medium [24].

To observe the dipole-mode vector solitons we used a photorefractive strontium-niobate crystal and the experimental set-up similar to that shown in Fig. 3 [25]. This time however, we used tightly focused circular beams (and a solid state laser operating at $\lambda=532 \mathrm{~nm}$ ). We applied two different methods to form the dipole structure. In the first instance we made use of the optical vortex formed by diffracting the laser beam on the computer-generated hologram phase mask. In this particular case we generated vortex with charge 1 (which corresponds to a $2 \pi$ angular phase shift). Both, vortex and Gaussian beams were then combined into a single beam and focused at the face of the photorefractive crystal. The dipole vector soliton was then formed as a result of modulational instability. In the second instance we prepared the dipole component by imprinting a $\pi$ phase jump across one of the beams by transmitting it through a glass slide. This beam was later combined with another Gaussian beam and focused onto the crystal. A CCD camera and set of imaging lenses were used to record the light intensity distribution on input and output facets of the crystal. Typical experimental results are shown in Figs. 6 and 7. Figure 6 illustrates the formation of the dipole-mode vector soliton via the break-up of the vortex beam. It has been known that the symmetry breaking instability causes a vortex beam to disintegrate when it propagates in the self-focusing isotropic medium [26]. The break-up fragments fly away with a nonzero tangential velocity which is a consequence of the initial angular momentum of the vortex beam. This effect has been observed in both, an isotropic nonlinear medium [27] as well as photorefractive crystals where inherent anisotropy speeds up the fragmentation process [28]. In the case of simultaneous propagation of the vortex and Gaussian beam the symmetry breaking instability is accelerated and results in the formation of a dipole-mode structure, as shown in Fig. 6c-d. A different situation is depicted in Fig. 7. This time the input beam is prepared in such a way that it mimics the structure of a dipole beam. The graphs in Fig. 6a show the initial intensity distribution for fundamental (u) and dipole (w) components. The graphs in Fig. 6b show the results of individual propagation of these two components. It is evident that the fundamental component forms a sin- 


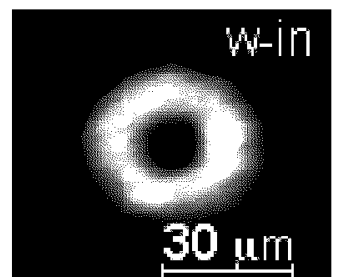

(a)

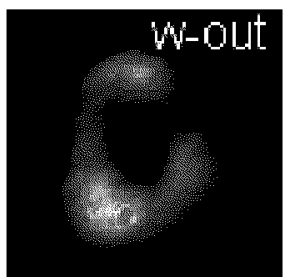

(b)

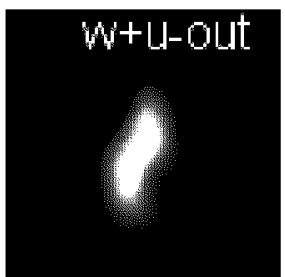

(c)

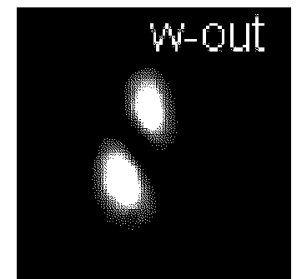

(d)

Fig. 6. Formation of a dipole-mode vector soliton via break-up of the vortex beam; (a) input intensity distribution of the vortex component; (b) break-up of the vortex component during individual propagation in the crystal; (c) output intensity of the dipole-mode soliton formed by co-propagating vortex and Gaussian beams; (d) intensity of the dipole component of the dipole-mode soliton. Parameters are: $V=2.3 \mathrm{kV}$, $P_{\mathrm{u}}=P_{\mathrm{w}}=0.3 \mu \mathrm{W}$, the propagation distance $z=6 \mathrm{~mm}$.
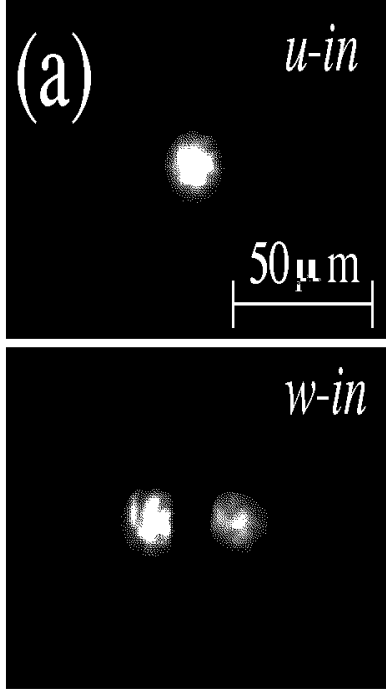

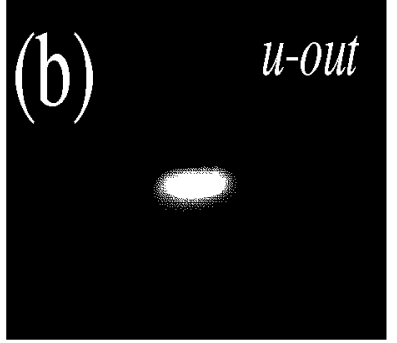

W-out

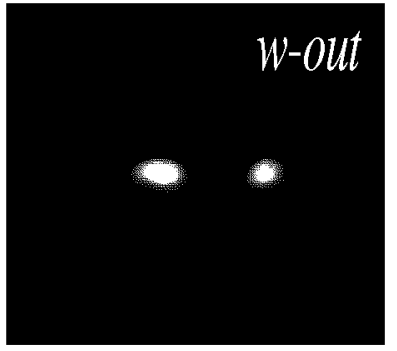

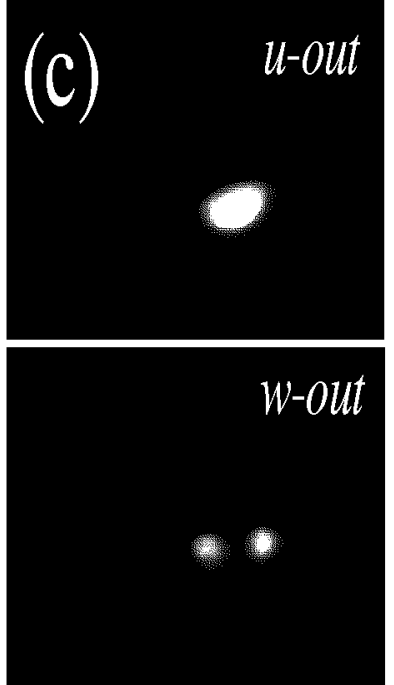

Fig. 7. Experimental observation of the formation of a dipole-mode vector soliton; (a) initial intensity distribution of fundamental (u) and dipole (w) components; (b) both components after an individual propagation in the crystal - the repulsion of the two out-of-phase lobes clearly visible; (c) simultaneous propagation of both beam components - the formation of a dipole-mode vector soliton. Parameters are: $V=1.8 \mathrm{kV}, P_{\mathrm{u}}=1 \mu \mathrm{W}, P_{\mathrm{w}}=2.6 \mu \mathrm{W}$, the propagation distance $z=10 \mathrm{~mm}$.

gle solitary beam while the dipole component splits into two fundamental solitons which fly away due to the repulsive force caused by the initial $\pi$ phase difference. Finally, the graphs in Fig. 6c show the result of the simultaneous propagation of both beams. It is clear that the presence of fundamental component leads to the appearance of an attractive force which prevents two repelling lobes of a dipole from escaping. Both beams propagate now as a stable dipole-mode vector soliton. 
The demonstrated here experimental realizations of the dipole-mode vector solitons opens up a possibility of further studies. These will include the stability of solitons, their collisional properties as well as the formation of more complex structures such as multipoles and "necklace" beams [29]. It should be mentioned that independent observation of the dipole-mode vector solitons has been reported by Carmon et al. [30].

In conclusion, we discussed multi-component vector solitons in an inertial nonlinear medium. These solitons are formed as a incoherent superposition of two or more components which correspond to fundamental and higher order modes of the self-induced waveguide. Both, one- and two-dimensional solitons have been observed in experiments with the photorefractive strontium-barium-niobate crystal.

I am grateful to my colleagues who contributed to the topic presented in this paper. In particular I am indebted to N. Akhmediev, A. Ankiewicz, G. McCarthy, C. Denz, M. Geisser, Y. Kivshar, B. Luther-Davies, E. Ostrovskaya, and C. Weilnau.

\section{References}

[1] see, e.g., a recent review on spatial solitons, G.I. Stegeman, M. Segev, Science 286, 1518 (1999).

[2] R.Y. Ciao, E. Germire, C.H. Townes, Phys. Rev. Lett. 13, 479 (1964).

[3] A. Snyder, J. Mitchell, Y. Kivshar, Modern Phys. Lett. B 9, 1479 (1995).

[4] M. Mitchell, M. Segev, D.N. Christodoulides, Phys. Rev. Lett. 80, 4657 (1998).

[5] D.N. Christodoulides, R.I. Joseph, Opt. Lett. 13, 53 (1988).

[6] D.N. Christodoulides, S.R. Singh, M.I. Carvalho, M. Segev, Appl. Phys. Lett. 68, 1763 (1996).

[7] A.W. Snyder, D.J. Mitchell, Phys. Rev. Lett. 80, 1422 (1998).

[8] J.U. Kang, G.I. Stegeman, J.S. Aitchison, N. Akhmediev, Phys. Rev. Lett. 76, 3699 (1996).

[9] N. Akhmediev, A. Ankiewicz, Solitons: Nonlinear Pulses and Beams, Chapman \& Hall, New York 1997.

[10] M. Mitchell, M. Segev, T. Coskun, D.N. Christodoulides, Phys. Rev. Lett. 79, 4990 (1997).

[11] D.N. Christodoulides, T.H. Coskun, M. Mitchell, M. Segev, Phys. Rev. Lett. 78, 646 (1997).

[12] V. Kutuzov, V.M. Petnikova, V.V. Shuvalov, V.A. Vysloukh, Phys. Rev. E 57, 6056 (1998).

[13] N.N. Akhmediev, W. Królikowski, A.W. Snyder, Phys. Rev. Lett. 81, 4632 (1998).

[14] A. Ankiewicz, W. Królikowski, N. Akhmediev, Phys. Rev. E 59, 6079 (1999).

[15] N. Akhmediev, A. Ankiewicz, Phys. Rev. Lett. 82, 2661 (1999).

[16] C.B. Manakov, Sov. Phys. JETP 38, 248 (1974).

[17] W. Królikowski, N. Akhmediev, B. Luther-Davies, Phys. Rev. E 59, 4654 (1999). 
[18] M.D. Iturbe-Castillo, P.A. Marquez-Aguilar, J.J. Sanchez-Mondragon, S. Stepanov, V. Vysloukh, Appl. Phys. Lett. 64, 408 (1994); M. Shih, M. Segev, G.C. Valley, G. Salamo, B. Crosignani, P. DiPorto, Electron. Lett. 31, 826 (1995); A.A. Zozulya, A.V. Mamaev, M. Saffman, D. Anderson, Phys. Rev. A 54, 870 (1996)

[19] N.M. Litchinitser, W. Królikowski, N. Akhmediev, G. Agrawal, Phys. Rev. E 60, 2377 (1999).

[20] M. Shih, P. Leach, M. Segev, M.H. Garret, G. Salamo, G.C. Valley, Opt. Lett. 21, 324 (1996).

[21] A.A. Sukhorukov, N. Akhmediev, Phys. Rev. Lett. 83, 4736 (1999).

[22] J.N. Malmberg, A.H. Carlsson, D. Anderson, M. Lisak, E.A. Ostrovskaya, Y.S. Kivshar, Opt. Lett. 25, 643 (2000).

[23] Z.H. Musslimani, M. Segev, D.N. Christodoulides, M. Soljačić, Phys. Rev. Lett. 84, $1164(2000)$.

[24] J.J. García-Ripoll, V.M. Pérez-García, E.A. Ostrovskaya, Yu.S. Kivshar, Phys. Rev. Lett. 85, 82 (2000).

[25] W. Królikowski, E. Ostrovskaya, C. Weilnau, M. Geisser, G. McCarthy, Y. Kivshar, C. Denz, B. Luther-Davies, Phys. Rev. Lett. 85, 1424 (2000).

[26] D.V. Skryabin, W.J. Firth, Phys. Rev. E 58, 3916 (1998).

[27] V. Tikhonenko, J. Christou, B. Luther-Davies, J. Opt. Soc. Am. B 12, 2046 (1995).

[28] Z. Chen, M. Shih, M. Segev, D.W. Wilson, R.E. Muller, P.D. Maker, Opt. Lett. 22, 1751 (1997).

[29] M. Soljačić, S. Sears, M. Segev, Phys. Rev. Lett. 81, 4851 (1998).

[30] T. Carmon, C. Anastassiou, S. Lan, D. Kip, Z.H. Musslimani, M. Segev, D. Christodoulides, Opt. Lett. 25, 1113 (2000). 